\section{Une nouvelle idée \\ du sacré: \\ le desir de santé \\ parfaite'}

\section{RESUMÉ}

Ce texte propose une comparaison entre les utopies classiques et c'est que l'auteur pressupose que soit la nouvelle utopie postmoderne. Selon lui, il faut signaler l'évolution contemporaine des utopies technologiques vers la science-fiction et indiquer qu'un genre est né: la sciencefiction utopiste.

\section{RESUMO}

Este texto propõe uma comparação entre as utopias clássicas e 0 que 0 autor pressupõe que seja a nova utopia pósmoderna. Segundo ele, é preciso assinalar a evolução contemporânea das utopias tecnológicas em direção à ciênciaficção e indicar o nascimento de um novo gênero: ciênciaficção utopista.

\section{MOTS-CLÉ (palavras-chave)}

- Utopie (utopia)

- Communication (comunicação)

- Science-fiction (ciência-ficção)

\section{Lucien Sfez}

Université Paris I/Panthéon-Sorbonne
CE N'EST QUE RÉCEMMENT que j'ai commencé à penser que je m'intéressais aux religions laïques.

Du temps où je m'occupais de la Décision ("Critique de la décision» Presses de Science Po, $1^{\text {ère }}$ ed. 1973, $4^{\text {ème }}$ ed. 1992), j'avais vu la prégnance du phénomène décisionnel, mais je le comprenais comme une idéologie de cadres et de managers. Quand plus tard, je me suis intéressé aux symboles en politique, j'ai bien compris la place centrale de "la politique symbolique » (PUF Quadrige $1^{\text {ère }}$ ed. 1978, $2^{\text {ème }}$ ed. 1993) et la présence du religieux en politique. Mais revenant bientôt aux idéologies de la post-modernité, j'envisageais la communication comme une idéologie encore ("Critique de la communication », Le Seuil, $1^{\text {ère }}$ ed. 1988, $3^{\text {ème }}$ ed. 1992), même si j'appelais la communication "religion des temps modernes ", mais ce n'était qu'une métaphore.

Ce n'est que lorsque j'ai fait l'analyse de la Santé parfaite ( La santé parfaite, critique d'une nouvelle utopie », Le Seuil, 1995) que j'ai compris qu'il s'agissait là non pas principalement d'une idéologie, mais d'une utopie. Sachant ici que j'avais lu Karl Mannheim² qui en 1929 avait opposé idéologie et utopie, puis s'était ravisé 30 ans plus tard et avait au contraire montré la continuité, les relations et l'interpénétration des deux champs. Reste qu'à mon sens dans cette sorte de ligne continue, il y a bien aux deux extrêmes deux pôles: L'utopie et l'idéologie, que ces deux pôles restent distincts et que appliqué à « la santé parfaite » cette idée signifie qu'elle est à la fois idéologique et utopique, mais bien d'avantage encore, utopique qu'idéologique.

En d'autres termes ici c'est le pôle 
utopique qui par sa force et son intensité l'emporte largement sur l'idéologie. D'autant qu'à cet élément utopique, s'ajoute désormais, selon moi, un élément de Science-fiction ces deux notes d'Utopie et de Science-fiction contribuent à créer la sonorité « sacrée » de la « santé parfaite »...

On traitera ici successivement d'abord des faits de l'enquête elle-même, menée aux Etats-Unis, au Japon et en Europe (I), puis de son traitement conceptuel (II).

\section{Histoires de voyage}

Je vais tenter de rapporter ici quelques éléments de l'enquête que j'ai mené aux EtatsUnis, au Japon et en France, suggérant au lecteur de se reporter, pour plus de détails et quelques approfondissements théoriques à mon livre La Santé parfaite, critique d'une nouvelle utopie (op.cit.). En 1990, après mes travaux sur la communication, je cherchais à déterminer quelle serait la prochaine idéologie dominante. Après deux ans d'errance je finis par localiser le phénomène : Les sciences du vivant en tant qu'organisatrices du sens dans nos sociétés dépourvues de référents. Je décidais donc d'une enquête portant sur le projet Génome et sur Biosphère II, duplicata en réduction de notre planète dans un hangar de verre, enfermée sur elle-même dans le désert de l'Arizona. Plus tard, en cours d'enquête, je fus conduit à un troisième objet : «Artificial Life».

\section{Projet Génome}

Dans les laboratoires de Boston, Washington, Seattle, Berkeley, Los Alamos, j'entendis des propos fort surprenants. On avait enfin trouvé (ou on allait trouver de façon imminente) le «Saint-Graal» de l'humanité. La cartographie et le séquençage du gène devaient nous donner les clefs de l'ultime détermination de la réalité et de la vérité. «Donnez-moi le génome d'un in- dividu, je vous dirai qui il est» proclamait le Nobel Walter Gilbert ignorant superbement l'interaction des gènes avec l'environnement, c'est-à-dire l'histoire et la culture. Il était urgent pour les scientifiques de nous débarrasser de nos «bad» gènes et de les remplacer par des «good» gènes pour obtenir un corps parfait. C'est ainsi qu'il y a des «bad" gènes, qui font de nous un jour des violents, des repris de justice, des alcooliques, voire des "homeless »(des sans-abris). Très surpris, je découvris ainsi ces premiers discours de purification générale : mais j'étais encore loin du compte.

Lors d'une visite rendue à Berkeley à des amis, j'en appris davantage. Voici des extraits de mon journal de voyage :

Emily, de Berkeley, psychologue, dont le mari est psychiatre. Couple chic, la soixantaine, qui connaît bien l'Europe, nos meilleurs vins et nos meilleurs fromages.

La discussion tourne toujours autour de la meilleure nourriture possible. Mais un jour, changement de cap : Emily nous informe qu'elle a subi une opération cet hiver. Etait-ce grave? Non, ça l'était virtuellement. Elle s'est fait enlever les ovaires... qui n'avaient rien. Mais sa mère et sa tante avaient eu un jour des ennuis aux ovaires. Il valait mieux les supprimer préventivement.

Devant ma stupéfaction («Jamais en France un chirurgien ne ferait cela», osais-je affirmer), Emily déclara «Il m'a fallu négocier avec les médecins. Ils m'ont fait promettre que s'ils m'enlevaient les ovaires, je ne supprimerai pas ensuite les seins». Je découvris alors que cette pratique de suppression préventive d'un ou deux seins était fréquente aux Etats-Unis, dans les «bons» milieux.

Le phantasme consiste ici à se purifier de ses organes, les uns après les autres. C'est dans ce contexte culturel 
qu'il faut songer à la pratique du dépistage génétique qui se développe actuellement en Californie. Les tests pré-nataux sont exigés par les lois. Quid si l'embryon est porteur de gènes défectueux ? La loi ne le dit pas encore. Mais les assurances le savent déjà. Elles refusent d'assurer ou assurent à des prix prohibitifs la santé de l'enfant à naître, plaçant la mère devant un dilemme : ou supprimer l'enfant, ou payer par elle-même durant sa vie entière. J'écris ces lignes au moment où le plan Clinton de sécurité sociale n'est pas encore adopté et où 35 millions d'américains ne sont pas couverts. Si ce plan est adopté, couvrira-t-il des cas de ce genre ? En d'autres termes, est-ce à la collectivité de payer pour le désir individuel de progéniture «défectueuse»? Le problème se posera sans doute en ces termes dans tous les pays bénéficiaires de la sécurité sociale, dont on sait déjà que le financement connaît des tensions insupportables.

En somme, quand Emily se fait enlever préventivement les ovaires, quand de nombreuses américaines se font enlever préventivement les seins, par crainte d'un capital génétique familial défectueux, on peut considérer qu'il y a là comme une anticipation. Lorsque le projet Génome sera plus avancé, lorsqu'on connaîtra avec précision les origines génétiques des principales maladies, le terrain sera déjà mûr pour la suppression des «bad » gènes. De la suppression des organes potentiellement dangereux à celle des mauvais gènes, il n'y a qu'un pas. Purification organique ou purification génétique, c'est tout un. Santé parfaite et utopie accomplie.

Quand je rapporte ces faits en France, on me regarde, étonné, un peu sceptique : N'est-ce pas là une affaire amé- ricaine, voire californienne ? Que non pas. En décembre 1995, deux mois après la sortie de mon livre, un Congrès international de médecine recommande, en cas d'ascendants à problèmes, de se faire couper préventivement seins et ovaires! (article de la revue Nature commenté dans Le Monde du 22 décembre 1995) Voilà déjà une utopie en marche. En voici maintenant une seconde: Biosphère II.

\section{Biosphère II}

Sous un hangar de verre, fermé, dont le seul contact avec l'extérieur est le soleil de l'Arizona nécessaire à la photo-synthèse, dans un lieu-dit nommé «Oracle» (je n'invente rien), on a recréé les cinq principaux biomes de l'humanité, installé 3.400 espèces végétales, quelques espèces animales, huit humains, quatre hommes et quatre femmes, durant deux ans. Ces humains respirent l'air produit par la végétation, se nourrissent des végétaux qu'ils cultivent et des produits des animaux qu'ils élèvent. Buts de l'opération : éviter la pollution de la terre et créer dans une Biosphère seconde (la première est la nôtre, celle où nous vivons) les conditions d'une purification totale de la planète, en même temps que prévoir par cette expérience un départ vers Mars (car les hommes vont un jour faire sauter la planète, à moins que la terre ne rencontre brutalement une étoile : il est urgent de partir).

Où l'on voit que la purification générale de la planète complète la purification totale du corps.

Ces deux purifications sont entreprises en même temps dans Biosphère II: le taux de cholestérol des humains enfermés est devenu très bas. Selon les critères médicaux, leur santé est supérieure à toute autre, elle est totale (au prix, il est vrai, d'avoir été soumis à la famine durant deux ans).

L'utopie donc continue. 


\section{Artificial Life}

Une troisième «expérience» complète les deux précédentes.

Christopher Langton et son équipe du Santa Fe Institute (Nouveau Mexique) créent des êtres artificiels dans l'ordinateur. Dotés d'un sexe, ils copulent, ont des enfants, se nourrissent (d'une nourriture électronique) attrapent des maladies, déclinent et meurent. Assemblés en troupeaux ils mènent aussi une vie sociale riche d'échanges...

«Ces êtres sont nos successeurs» dame Langton. «Nous sommes des Dieux» ajoute-il, «nous qui pouvons créer nos propres successeurs», électroniquement parfaits et même si l'on veut immortels («je peux vous les créer immortels» me dit Langton ; «je n'en doute pas, je n'en doute pas» répondis-je).

Le but de l'Artifidal Life est un but de transformation totale de l'humanité. Son telos est de créer un être supérieur, une autre forme de vie. C'est là l'horizon limite de l'utopie de très «Grande Santé». Euvre alchimique, bien au-delà de la «petite» intelligence artificielle. Grand rêve démiurgique que celui qui consiste à engendrer ses successeurs. Au prochain siècle (nous y sommes pratiquement) ce sera chose faite ${ }^{3}$. Ces êtres auront un jour une conscience, une volonté, une âme ${ }^{4}$. Un auteur, pourtant sceptique, ne dit-il pas qu'au moins les robots semblent s'organiser eux-mêmes, à l'aveugle, et selon un mode de sélection naturelle ${ }^{5}$ ? A tel point qu'une des principales préoccupations de Langton est de créer une éthique adaptée à ces êtres là. " Il y a d'autres vies que les nôtres qui ont droit à l'existence. D'ici 10 ans nous construirons les machines auto-organisées. Aura-t-on le droit de les mettre à mort à notre gré ? Quelle serait notre légitimité à le faire ? Mais si elles commettent des crimes ou des fautes, quelle punition infliger ? La mort, la mutilation, la prison ? » Et devant notre scepticisme il nous répète : " Mais c'est pour dans 10 ans. Il faut s'y préparer. $»^{6}$.

Nous voilà loin des rafistolages artisanaux et mesquins de la thérapie génique : il s'agit ici d'êtres électroniques, immortels et parfaits. L'entreprise de purification générale est totalement accomplie. Purification du corps individuel (projet génome) dans une planète purifiée (Biosphère II) : le tout surplombé par les êtres électroniques, produits des Dieux que nous sommes devenus, et petits dieux eux-mêmes. Le devoir de bonheur, devoir de purification globale s'accomplit enfin dans "la Santé Parfaite $»^{7}$.

\section{Le traitement conceptuel}

Sans doute existe-t-il ici des éléments idéologiques qui ne sont pas négligeables: Je songe ici à cette idée étrange de la recherche dans des laboratoires américains très sérieux, du gène des repris de justice, des violents ou des "homeless» (les sansabris) car cette société américaine auto-gratificatrice ne peut supposer un seul instant que devenir un sans-abri ne puisse avoir d'autres raisons que génétiques. Je songe ici encore aux abus, idéologiquement marqués de la théorie de l'information, là où elle ne devrait pas avoir droit de cité. Sur ces deux points idéologiques, je renvoie bien sûr à "la santé parfaite » (op. cit.). Mais la santé parfaite est surtout une utopie car sa force spécifique réside dans son caractère utopique, dans la prégnance de son utopie, sans compter bien sur la science-fiction qu'on va présenter maintenant.

\section{A La santé parfaite est ułopique}

1 Un trajet : Des utopies classiques aux projets utopiques actuels.

Les utopies classiques

D'après la forme des récits utopiques que nous avons pu analyser, on peut dégager 
cinq marqueurs du texte utopique.

\section{1/ Le lieu isolé du récił}

Quel est donc la situation de ce lieu? C'est un lieu clos, clôturé tant par ce qui est dit de ses frontières, montées en défense, que par le récit lui-même qui isole le fictif du réel. Cet espace n'est pas territorial, même si des précisions de type géographique sont données au cours de la narration.

La tabula rasa est alors complète, nous voici de l'autre coté du monde. L'île rebaptisée, la langue réinventée.

L'île étant la forme parfaite de la clôture, le récit utopique la sollicite le plus souvent. D'autant que l'île accrédite la fable selon laquelle un voyageur, égaré, y échoue et se met à la décrire.

L'île ainsi préservée de toute contamination, est défendue contre l'extérieur, ce ne sont que digues, remparts, fossés ou bien encore océans tumultueux et rivages inabordables. Si le voyageur, personnage indispensable de la fiction, y aborde, il sera procédé à une enquête sur sa moralité et il devra passer un test avant d'être accepté. Dûment endoctriné, il pourra enfin être admis. Isolement, fermeture, espace marqué, défense des frontières, tels sont les caractè-

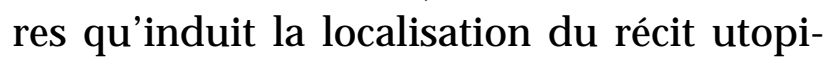
que dans l'île.

\section{2/ La toute puissance du narrateur}

Un voyageur, généralement honnête homme, découvre cette terra incognita, et, de retour dans son pays, raconte ce qu'il a vu.

Evidemment le narrateur, ou récitant, est le double de l'écrivain, qui cependant se met en jeu face à lui, cette fois comme auditeur.

Avec ce dispositif, le caractère fermé et autoritaire de l'utopie, déjà présent par la localisation îlienne, est renforcé puisque il n'y a en définitive qu'un seul maître du jeu : celui qui produit les règles. Dispositi- on qui trouve son écho dans la hiérarchie des fonctions attribuées aux personnages de la fable : le Directeur, le Gouverneur, le Légiste. Il en est ici comme pour l'île, illustration de la structure même du récit, l'auteur, maître absolu de sa fiction, est présenté à l'intérieur de la narration par les instances de pouvoir.

\section{3/ Des règles de vie hygiénistes}

Dans cet univers clos, le travail, les loisirs, la santé, la justice sont réglés au quart de tour. Ces régies et ce qu'elles règlent varient selon les auteurs, et selon les préoccupations du siècle. Mais elles sont toujours là, imposées, étroitement surveillées. Toute infraction est sévèrement punie. Déjà, à l'entrée, un filtrage. Dans l'Abbaye de Thélème les habitants doivent être beaux, bien faits et aimer les plaisirs. Pour accéder à l'Icarie, de Cabet, le voyageur (encore un!) Lord W. Carisdall, subit trois mois d'épreuves dans une ville intermédiaire. Seuls les bons éléments peuvent entrer. A ce filtrage, correspond, à l'intérieur, une sorte d'eugénisme tantôt doux, tantôt violent. Le XIXème siècle est féru d'hygiène, de santé physique et morale, et donc de répressions. On voit même dans l'Icarie de Cabet l'interdiction du tabac, pour raison de santé... les aliments sont votés, on décrète «l'inscription d'un nouveau légume sur la liste des aliments». La loi exige chasteté et pureté, et éloigne toute tentation d'adultère (puni de mort par exemple chez Burton, ou femmes flagellées sur la place publique chez Vairasse, dans Histoire des Sévarambes ). La propreté des corps est aussi la propreté des âmes, aucune souillure ne doit compromettre l'ensemble. On verra ainsi chez Jules Verne, dans «Les 500 millions de la Begum», la cité de France-Ville se soumettre à l'impératif du nettoyage : «Lavez, lavez...» devient un mot d'ordre. Rectiligne, géométrique, redressé s'il est besoin, le territoire est quadrillé, aplani, distribué en lots égaux. 


\section{4/ L'imaginaire technique}

La technique a toujours été sollicitée par les utopies, comme le Deus ex machina qui rend possible des dispositifs singuliers.

A ce titre, l'utopie compte sur la mécanique - celle des lois de distribution des richesses pour Cabet, celle de l'énergie pour Villiers de l'Ile Adam - pour faire advenir d'un coup ce que les choses laissées à ellesmêmes mettraient sans doute, dans l'ordre du réel, des siècles à accomplir. L'utilisation de la technique assure donc au récit la possibilité de transformations immédiates, elle est un ressort indispensable à l'allure du récit.

Utopus fait « couper » l'isthme qui relie Utopia à la péninsule Abraxa. Comment? Ce n'est pas dit. La «technique» s'en charge.

La technique ainsi revendiquée instaure donc un monde à son image. Le hasard en est exclu, car le hasard fait partie du monde réel, naturel, que l'utopie tient justement à distance.

Monde sans aléa, sans impureté, sans mort ni décomposition, sans poussières ni étrangetés, sans jeux ni plaisirs, et sans autre respiration que celle qui commande l'ensemble de la grande machinerie.

Monde, en ce sens, sur-naturel et qui doit sa supériorité à l'artifice.

La technique joue donc le double rôle d'une aide structurelle pour le récit, au niveau du texte, et d'un leitmotiv interne au contenu idéel de l'utopie.

\section{5/ Le refour à l'origine}

On comprend alors le tubula rasa: Il faut se débarrasser de ce qui existe pour retrouver ce qui est.

On voit par là-même que l'état sauvage, la barbarie sans langage et sans écriture, ne peuvent représenter la vérité de la nature : Elles n'en sont que la pré-Histoire, attendant d'être re-formées. Les voyageursmissionnaires du XVIIIème rencontrant les
Indiens n'ont qu'une hâte, celle de les instruire. Et pour cela, le récit fictif et ses marqueurs passant dans les actes, ils appliqueront à ces peuplades désordonnées les recettes du récit utopique.

Re-nommer : Les sauvages reçoivent à la fois baptême et nouveau nom.

Re-éduquer la langue: Il faut leur apprendre à parler la langue convenable.

Reconstruire les villages, après les avoir détruits, selon les règles de la géométrie, effaçant ainsi, d'un coup de technique, les fondements structuraux des sociétés basées sur le marquage au sol, comme le montre Levi-Strauss avec les Bororos.

Un tournant : l'utopie technologique américaine

Cet élément significatif, cette valeur qui intégrait les acquis culturels de l'Europe mais devait marquer aux yeux des Américains leur différence et donc leur identité en tant que nouvelle nation, ce fut la technique.

Autour de la technique, de ses inventions, de son pouvoir de transformer la vie, se bâtit le rêve américain, partagé par tous. Une identité se construisait, et les fictions utopiques servirent la cause de cette identité. Il ne s'agissait plus de re-fonder, de critiquer, mais de fonder tout simplement. Le ton changeait, il passait de la négation (outopos : le non-lieu) à l'affirmation remplie d'allégresse d'un lieu fabuleux mais réel : l'Amérique.

Le mouvement des utopies classiques qui, du réel allait vers le fictif et faisait entrevoir des mondes dont le lecteur savait qu'ils étaient irréalisables, s'inverse dans les utopies technologiques américaines. Ce que la fable fait miroiter, c'est la réalité prochaine, ce dont elle parle, c'est d'une réalité présente. Entre le présent et l'avenir le récit tisse l'histoire, certes imaginaire, mais crédible, du progrès nécessaire des techniques.

Entre 1883 et 1933 on note 160 «tech- 
nological» utopies dont 35 seulement sont à peu près connues, mais dont l'une au moins eut un succès foudroyant, autant qu'imprévu «Looking backward» d'Edward Bellamy (1888) eut des millions de lecteurs. Les auteurs utopiques à la différence de leurs confrères européens sont souvent ingénieurs civils, dans l'aéronautique et la mécanique, hommes d'affaires, industriels, ou administrateurs d'entreprises, architectes ou télégraphiste... Ils écrivent des manuels techniques, quelques-uns même sur la cuisine. En somme écrire une utopie est une occupation en phase avec leur métier et reflète leurs confiance et leurs croyances en un avenir technologique.

Ce qu'il faut remarquer ici c'est, comme le dit Howard Segal, qu'il y a peu de différence entre les utopies écrites, les récits utopiques, et les utopies non-fictionne$1 l e s^{8}$. Autrement dit le passage d'un genre (le récit) à l'autre (l'action) ne révèle aucune différence dans la sorte de vision qui s'y exprime.

Plans, programmes, planifications, tentatives de fonder des communautés, associations de toutes sortes, naissent et se développent en accord avec les ouvrages utopiques, accompagnés ou préfigurés par les récits utopiens. Ce n'est que «scientific management » de toutes parts, une véritable "utopian industrial army » s'attaque à toutes sortes de problèmes comme la fonctionnalisation des écoles, du gouvernement, de la justice, de la médecine, des entreprises, des travailleurs, etc... La prolifération des organisations ne le cède en rien à la prolifération des écrits utopiques.

\section{Les trois projets Génome, Biosphère II et Artificial Life sont utopiques.}

Les deux projets Génome et Biosphère II s'inscrivent dans une visée utopique, non point en ce qu'ils ne seraient pas réalisables - ils sont en effet en voie de réalisation et bien engagés - mais en ce qu'ils partici- pent tous deux d'une projection de plusieurs éléments idéologiques sur l'avenir de l'humanité et de la planète. Eléments que chaque projet lie de façon à former un tout universalisable.

Les marqueurs de l'utopie sont alors concrétisés en dispositifs singuliers que l'on peut expérimenter, tandis que leurs origines fictionnelles sont, elles, bien reconnaissables. Il en est ainsi des principales caractéristiques du récit :

L'isolement : La biosphère, de forme circulaire, est amarrée dans le désert de l'Arizona comme une île, offrant sa transparence de verre intraversable aux visiteurs, tandis qu'elle protège les biosphèriens, nouvelle race d'hommes. Les laboratoires de génétique sont tout aussi bien des forteresses, défendues par les chercheurs contre l'ennemi potentiel, prêt à en voler les secrets ou à les exploiter (l'histoire des brevets en fait foi).

La toute-puissance du narrateur: Celui-ci est ici devenu pluriel et anonyme et se fond dans une collectivité. C'est cette collectivité, elle, qui parle, transmet au dehors, publie et débat. Pour les publics non-spécialisés que nous sommes, pour la plupart, la parole des comités et associations de chercheurs est incontestable, ils sont maîtres de leur « récit », c'est-à-dire de leurs travaux, protégés par le statut non plus de littéraires mais de savants.

L'imaginaire technique est devenu la substance même de la recherche et non plus une aide à l'intrigue. Sans la technologie toutepuissante, les projets eux-mêmes n'auraient pu avoir lieu. Bien qu'il soit gommé souvent par les scientifiques "purs", le rôle des machines est primordial. Elles sont acteurs à part entière.

Les règles de vie hygiéniques sont surtout repérables pour Biosphère II. Appliquées strictement à l'intérieur de la bulle, elles sont exemplaires, c'est-à-dire doivent, selon les biosphèriens, devenir la règle universelle 
pour tous les hommes. Les écologistes, sorte de Biosphèriens sans bulle, ne sont pas plus avares de leurs recommandations, et pour les hommes, et pour la Terre. Moins aisément discernables pour la communauté des généticiens, ces règles de vie parfaite constituent la finalité des travaux. Et les moyens d'y parvenir sont établis comme préceptes: Une fois débarrassée des mauvais gènes, c'est une race nouvelle, obéissant aux principes régulateurs du «bon» génome, qui naîtra.

Enfin, le retour à l'origine hante les deux projets. C'est une origine rêvée, d'avant les maladies de la société, une origine pure, où nature et artifice humain seraient liés, formant un tout d'avant la séparation. Ce tout matière/esprit prend la forme d'une technosphère dans la version athée ou noosphère dans la version spiritualiste.

Et ceci sans compter l'extraordinaire "Artificial Life» qui nous prive de corps (purification totale) mais aussi, dans cette entreprise forcenée de transparence et d'hygiène, nous prive aussi de notre esprit, puisque nous ne sommes plus que des robots électroniques. Ici encore les cinq marqueurs fonctionnent admirablement.

\section{B La Santé parfaite relève aussi de Ia Science-Fiction}

Ce que préparait "Artificial Life » se retrouve impeccablement et porté à un niveau supérieur dans "souls in silicon » ou le passage vers la surhumanité.

"Ames dans la Silice.»

Tel est le titre d'un article paru dans la revue $\mathrm{OMNI}^{9}$.

$C^{\prime}$ est une histoire et son commentaire. Elle commence en 2050 environ. Il s'agit de vous. Vous avez 90 ans, vous êtes donc encore un jeune homme en ces temps où la médecine a triomphé de presque tout. Votre médecin vous dit, après quelques analyses, qu'il a de mauvaises nouvelles à vous apprendre. Vous allez mourir dans les six mois. Vous êtes l'un des rares cas où la médecine ne peut rien. Vous êtes, bien sûr, consterné de quitter votre femme, vos amis, dans la fleur de l'âge... Mais le docteur ajoute : «Vous avez une alternative». Ah ! L'espoir revient. Vais-je vivre ? Non, pas exactement. "Votre corps va certainement mourir. Mais vous êtes un candidat pour la transplantation de l'esprit».

Deux semaines plus tard, l'opération se déroule sous vos yeux, en toute conscience. L'anesthésie n'a concerné que la peau du crâne, car le cerveau, lui, non innervé ne souffre pas. Vous pouvez vérifier ce que font les médecins sur un écran de télévision. Ils vont détacher le cerveau et son corps calleux et le placer dans un ordinateur.

L'opération est terminée. "Félicitations», vous dit le médecin. "Bienvenue en immortalité». Votre chance est inouie. Au début, l'ordinateur sera un auxiliaire de votre cerveau. Le cerveau lui donne toute sa mémoire. L'ordinateur l'accélère et vous pouvez réciter à votre femme avec la plus grande précision la recette du poulet que vous mangeâtes avec elle il y a trente ans à Paris. Vous êtes, aussi, une très grande mémoire (accélérée et précise) des moindres recoins de votre entreprise et vous devenez donc un manager encore plus précieux. Vous trouvez d'ailleurs que les esprits "vivants», vivant avec leur corps, sont un peu trop lents.

Vous préférez dialoguer avec d'autres immortels comme vous, parce qu'avec eux ça va plus vite. Un jour votre cerveau organique se détruit (comme toute chose organique). Mais quelle importance ? Votre esprit continue de vivre éternellement dans l'ordinateur, avec ses souvenirs, ses procédures, ses préférences et ses manies.

Direz-vous que cela ne peut exister sans corps, sans votre corps disparu ? Faux. Car souvenez-vous de Stephen Hawking, le grand physicien contemporain, atteint d'une dégénérescence prononcée (appelée ACS ou maladie de Lou Gehrig) dont on n'entend même plus la voix et qui s'exprime par ordinateur et par une voix 
synthétique. Il a même trouvé le moyen de divorcer de sa femme pour épouser son infirmière...

Vous pouvez donc être vous-même sans votre corps. Tout votre appareil sensible sera dans les sensors électroniques de votre ordinateur. Mais avez-vous autant besoin que vous le croyez de sensibilité extérieures ? Que non. Voulez-vous un plaisir sexuel ? Pas besoin de partenaire. Vous excitez certains points de votre cerveau qui contiennent l'interprétation du plaisir sexuel. Même chose pour la nourriture. Et vous pouvez programmer les plus merveilleux voyages en Europe ou en Orient. D'ailleurs mieux encore, vous pouvez tout imaginer. Lorsque vous étiez enfant, vous regardiez les films à effets spéciaux de Georges Lucas, en sachant que ce n'était pas la réalité, mais des images fabriquées à partir d'ordinateurs. Ces techniques sont arrivées aujourd'hui en 2050 à la perfection. Vous pouvez vous «brancher» sur des machines à effets spéciaux, en imaginant toutes sortes d'aventures.

Voilà pour les distractions.

Quant au travail, vous êtes délivré d'un corps perdu de maladie et vous êtes plus efficace que jamais. Si bien que lorsqu'un représentant de votre entreprise vient vous voir, à l'occasion de votre centième anniversaire, pour vous suggérer avec précaution $q u^{\prime}$ il serait temps de prendre votre retraite, vous le regardez (c'est une femme) avec commisération car vous vous rendez compte qu'elle commence vraiment à accuser son âge et vous lui dîtes : «Non, merci. Je vais vous quitter. Je suis recruté par la NASA qui a besoin de moi pour ses vols intersidéraux. Ils préfèrent un esprit sans corps. Car un esprit avec corps est plus difficile à gérer dans l'espace. Et, par ailleurs, ils veulent un esprit humain capable de prendre des décisions appropriées, devant des situations imprévisibles, et non pas un simple ordinateur». Une nouvelle carrière vous attend. Juste à la fin de votre second centenaire.

Avons-nous besoin d'en rajouter? Cet- te nouvelle se suffit à elle-même et résume la totalité du propos. Nous sommes dans le droit fil de la perfection d'Hadaly, héroïne de «L'Eve future » de Villiers de 1'Isle Adam. Il s'agit de vous, de moi. Je-nousGaia, comme dit une autre héroïne, celle d'Asimov. Tout y est : la liaison la plus amicale de l'homme et de la machine, un corps enfin maîtrisé (une nourriture qui donne du plaisir sans être ingérée, un sexe sans sexe, c'est-à-dire sans sida et sans grossesse, des voyages sans fatigue, plus de cholestérol et plus de cancer), une idéologie de l'efficacité à toute épreuve au travail ou en famille, le voyage dans l'espace comme accomplissement de l'homme : autant de thèmes déjà repérés dans le projet Génome, dans Biosphère 2 et dans 1'Artificial Life. La perfection totale et l'immortalité dominent l'ensemble, sont à la fois le but poursuivi et les moyens qui le réalisent.

Merveilleuse nouvelle qui dit le tout de l'imaginaire technicien de l'An 2000. Jusqu'au tautisme, néologisme que j'avais créé dans "Critique de la Communication » (op.cit.) par contraction de autisme et de tautologie pour situer la maladie qui nous vient de l'écran qui nous sidère, nous rend sourd-muet par ses répétitions mêmes qui sont le mode habituel d'administration de la preuve du monde journalistique et du monde politique.

\section{Notreconclusion : une Science-fiction utopiste}

Au-delà de Saint Simon, il nous faut signaler l'évolution contemporaine des utopies technologiques vers la science-fiction et indiquer qu'un genre est né ${ }^{10}$ : la science-fiction utopiste (ou SFU ), qu'on peut résumer par deux traits :

Une compression du temps. En effet, au lieu de projeter une image d'avenir à partir du présent, la SFU construit l'avenir à partir d'un passé hypothétique (le dinosaure) immédiatement raccordé au futur. En effet, 
si le présent peut prêter à contestation, ni un passé aussi lointain, ni un avenir tout aussi lointain ne peuvent être jugés sur pièces : ils restent donc tous les deux illusoires, mais s'entretiennent mutuellement de leur vraisemblance. Ce qui compte pour la SFU c'est cette hésitation entretenue entre rêve et réalité : si les dinosaures ont existé, ce qui est incontestable, on ne connaît pas cependant leurs conditions concrètes de vie et de mort, alors la réalité virtuelle vient à se substituer à notre ignorance. Les deux extrêmes du temps, passé et futur, se rejoignent dans un même sentiment de demi-croyance. Le présent a dispa$\mathrm{ru}$, « compacté » en quelque sorte dans un temps dont la durée vécue est absente.

Une hybridation d'images. Les figures du discours qu'employaient les utopies classiques, ont acquis avec les outils technologiques une sorte de réalité : elles sont visibles, elles sont des images d'abord, auxquelles le texte est surajouté (alors que dans l'utopie classique l'image venait à l'esprit du lecteur d'après le texte) elles ne peuvent donc, pense-t-on, tromper.

$C^{\prime}$ est un lieu commun que de constater l'emprise et l'empire de l'image sur notre monde contemporain. Mais il faut bien comprendre les effets de cet entassement d'images : il installe un troisième monde entre le réel et l'imaginaire, un " demimonde ${ }^{11}$ si l'on veut, et où il est impossible ou du moins difficile de faire le départ entre nature et artifice (les tamagotchis en sont un exemple). Nous vivons alors dans la fiction, dans un univers mi-apparence mi-réalité ; films, téléfilms et documentaires aidant, nous ne démêlons qu'à grand peine ce qui appartient à la science ou à la fiction, au réel ou à l'illusion. Le monde du Net, devenant le monde tout court et non plus un outil pour opérer sur le monde, projette son illusion sur l'ensemble de nos croyances. Tout l'appareillage technique du virtuel, en vérité fort compliqué, au lieu de faire obstacle à notre croyance sert plutôt à renforcer l'impression que ce virtuel est à notre portée. En fait, même si par ail- leurs un certain bon sens nous dictait la prudence, le vraisemblable est à la mesure de ce que nous voulons croire •

\section{Notas}

1 Voir Lucien Sfez "La Santé Parfaite", Le Seuil, 1975; Trad. Bresilienne, éditions Loyola, preface de Roberto Motta, anthropologue à Recife.

2 Voir Karl Mannhein, "Idéologie et Utopie", Marcel Rivière, 1956.

3 Voir Steven Levy, "Artificial Life", Pantheon Books, 1992, p.344.

4 Ibidem, p.340 et sq.

5 "There is still something mysterious" Roger Penrose in The Emperor's new mind. Oxford University Press, 1989 , p.416.

6 Interview au Santa Fe Institute, du 10 octobre 1992.

7 Titre de notre ouvrage, Op. cit.

8 Howard Segal, "Technological Utopianism in American culture", University of Chicago Press, 1985.

9 Novembre 1993, «Souls in silicon» par Frederick Pohl et Hans Moravec, p.67.

10 Voir par ex : «Etonnants voyageurs, utopies S.F.», Anthologie présentée par Michel Le Bris avec le collaboration de Jacques Chambon, Hoëbekeim, 2000. Dans ces textes c'est visiblement la technique qui fait le passage de l'utopie vers la science-fiction. Dans le même sens voir «Utopie et science-fiction » par Serge Lehman, Le Magazine Littéraire, mai 2000, p.31.

11 Et ceux qui le pratiquent sont des «demi-mondains» 